\title{
Local kinetic interpretation of entropy production through reversed diffusion
}

\author{
A. Porporato* \\ Department of Civil and Environmental Engineering, Duke University, Durham North Carolina, USA \\ P. R. Kramer \\ Department of Mathematical Sciences, Rensselaer Polytechnic Institute, Troy, New York, USA \\ M. Cassiani \\ NILU - Norwegian Institute for Air Research, NO-2027, Kjeller, Norway \\ E. Daly \\ Department of Civil Engineering, Monash University, Clayton, Victoria 3800, Australia \\ J. Mattingly \\ Department of Mathematics, Duke University, Durham North Carolina, USA \\ (Received 20 July 2011; published 31 October 2011)
}

\begin{abstract}
The time reversal of stochastic diffusion processes is revisited with emphasis on the physical meaning of the time-reversed drift and the noise prescription in the case of multiplicative noise. The local kinematics and mechanics of free diffusion are linked to the hydrodynamic description. These properties also provide an interpretation of the Pope-Ching formula for the steady-state probability density function along with a geometric interpretation of the fluctuation-dissipation relation. Finally, the statistics of the local entropy production rate of diffusion are discussed in the light of local diffusion properties, and a stochastic differential equation for entropy production is obtained using the Girsanov theorem for reversed diffusion. The results are illustrated for the Ornstein-Uhlenbeck process.
\end{abstract}

DOI: 10.1103/PhysRevE.84.041142

PACS number(s): 05.40.-a, 05.20.-y, 05.70.Ln

\section{INTRODUCTION}

Stochastic diffusion processes are used to model a variety of phenomena, from Brownian particles and macromolecules to turbulent dispersion and economic and environmental fluctuations [1-5]. Several theoretical and practical problems require reconstructing backward in time either the whole process or some specific trajectories thereof (e.g., in nonequilibrium statistical mechanics [6-15], control theory [16,17], and turbulent dispersion [18-21]). Related issues appear in reciprocal diffusion and Brownian bridges [4,5,22,23], as well as in the probabilistic interpretation of quantum mechanics, in relation to the so-called Schrödinger problem [22-31] of finding the stochastic dynamics that "interpolates" between a pair of given probability distributions at two different times. With regard to the statistical mechanics of nonequilibrium systems, recent developments (e.g., $[10,14]$ ) have shown that the local entropy production can be expressed as the relative entropy between forward and backward velocities [6,10-15]. Since thermal fluctuations can be modeled as Gaussian noise, detailed analysis of stochastic diffusion is thus of great interest.

The purpose of this work is to discuss some fundamental kinetic quantities of diffusion processes, in particular the mean local velocities (e.g., drift, current, osmotic, and thermal) and acceleration, and their behavior under time-reversal. Local kinematic and dynamic properties are essential for a microscopic description of diffusion, but their definition and

*amilcare@duke.edu analysis require particular attention because of infinitely fast fluctuations of the white-noise forcing [1]. While numerous papers have explored such links before, the angle from which we view these topics is complementary to previous works and draws connections between nonequilibrium statistical mechanics (e.g., [6-15]) and stochastic mechanics and reciprocal diffusion (e.g., [22-31]). Our hope is that a holistic treatment of the subject will help improve intuition. The insights so obtained are linked to the hydrodynamic description, the Pope-Ching formula for the stationary distribution [32], and expressions for the rate of entropy production.

We discuss the time reversal of free (or uncontrolled) diffusion, in the presence of multiplicative noise and from the standpoint of two stochastic calculi (Itô and HänggiKlimonotovich) [33-35]. From a thermodynamic viewpoint, the multiplicative noise corresponds to inhomogeneous temperature distributions [36], whose nonequilibrium behavior is known to be only partly captured by a white-noise (i.e., Smoluchowski), local-equilibrium approximation [37]. Within this approximation, the kinetic (Hänggi-Klimontovich) noise prescription has the advantage that its drift naturally incorporates the effects of the thermophoretic force due to inhomogeneous temperature distributions. For simplicity we restrict our analysis to one-dimensional problems.

Section II reviews the Itô and Hänggi-Klimontovich interpretations of stochastic differential equations and relates their coefficients to the entropy production rate, while Sec. III develops the equations for time-reversed diffusion, and Sec. IV relates local velocity and acceleration to the forward and backward diffusion coefficients. Section V draws 
the connections between entropy production and the local velocity and acceleration of the forward and reversed diffusion processes. Finally, Sec. VI illustrates the ideas on the Wiener and Ornstein-Uhlenbeck processes, and Sec. VII summarizes our conclusions.

\section{FORWARD DIFFUSION}

Consider a diffusion process evolving forward in time [2], described by the stochastic differential equation (SDE) in the Itô form

$$
d X=a(X) d t+b(X) d W(t)
$$

where $d W(t)$ is the increment of the Wiener process (white Gaussian noise) evolving in time $t$, and $a(X)$ and $b(X)$ are the drift and diffusion terms, respectively, here assumed to be time-invariant for simplicity. The state dependence of the diffusion term $b(X)$ is often referred to as multiplicative noise. The probability density function (PDF) of $X$ evolves according to the Fokker-Planck (FP) equation,

$$
\frac{\partial p}{\partial t}=-\frac{\partial}{\partial x}(a p)+\frac{1}{2} \frac{\partial^{2}}{\partial x^{2}}\left(b^{2} p\right)
$$

where $p$ may refer to either the transition PDF, $p\left(x, t \mid x_{0}, 0\right)$, with initial condition $\delta\left(x-x_{0}\right)$, or the one-time PDF, $p(x, t)=$ $\int p_{0}\left(x_{0}\right) p\left(x, t \mid x_{0}, 0\right) d x_{0}$, with generic initial condition $p_{0}(x)$ [2]. We will assume that a transient solution exists for the free process for any time $t$ until an arbitrary time $T(0 \leqslant t \leqslant T)$, which will be the origin of the reversed time, $\tau=T-t$.

The Itô interpretation of the noise requires interpreting Eq. (1) in a causal way [2], whereby the coefficient of the noise is evaluated at the beginning of the time increment under consideration:

$$
X(t+\Delta t)-X(t)=a(X(t)) \Delta t+b(X(t)) \Delta W(t) .
$$

Accordingly, one can show [2] that $\langle\Delta X\rangle=a(x) \Delta t$ and $\left\langle\Delta X^{2}\right\rangle=b^{2}(x) \Delta t+o(\Delta t)$ for small $\Delta t$, where $\Delta X$ is computed in a causal or forward way:

$$
\Delta X=\left.(X(t+\Delta t)-X(t))\right|_{X(t)=x} .
$$

As a result, the local forward velocity $\Delta X / \Delta t$ is a Gaussian random variable with mean $a$ and variance $b^{2} / \Delta t$ for $\Delta t \rightarrow 0$ [2] (for simplicity, we will often dispense with writing explicitly the arguments of the various averaged quantities).

We also consider the (acausal) kinetic HänggiKlimontovich (HK) prescription [33-35], for which the noise coefficient is evaluated at the end of the discretized time interval of interest. A stochastic differential equation to be interpreted in this sense will be written

$$
d X=h(X) d t+b(X) \star d W(t)
$$

which is to be understood as the $\Delta t \rightarrow 0$ limit of the discretization,

$$
X(t+\Delta t)-X(t)=h(X(t)) \Delta t+b(X(t+\Delta t)) \Delta W(t) .
$$

This prescription is equivalent to Eq. (1) if we take

$$
h=a-b \partial_{x} b .
$$

In general, $h$ is a combination of the first- and second-order moments of $\Delta X$ and is equal to $\langle\Delta X\rangle / \Delta t$ only in the case of additive noise. The kinetic prescription gives rise to the transport form of the Fokker-Plank equation,

$$
\frac{\partial p}{\partial t}=-\frac{\partial}{\partial x}(h p)+\frac{\partial}{\partial x}\left(\frac{b^{2}}{2} \frac{\partial p}{\partial x}\right),
$$

where $b^{2} / 2$ is the so-called diffusivity. Such noise interpretation has been used, albeit without explicit mention, in previous works dealing with thermodynamic interpretations of stochastic diffusion [7,8,13].

As also discussed in [38], when modeling the stochastic dynamics of Brownian particles, drift and diffusion have to be consistent with a fluctuation dissipation relation, and this is more naturally seen in the HK prescription [see, e.g., Eq. (71) later in this paper], which gives Eq. (8) and the exponential form of the PDF (canonical Boltzmann distribution). This has to do with the fact that when a particle is immersed in a nonhomogeneous environment, the temperature distributions of the bath and the particle properties are imposed and these, in turn, determine the drift and the diffusion terms of the Brownian particle. The particular form of the stochastic calculus chosen in the SDE becomes then a matter of formal convenience, as long as the results are consistent with the underlying physics.

\section{A. Hydrodynamic description and local entropy balance}

The previous forward FP Eqs. (2) and (8) can be rewritten in hydrodynamic form as a continuity equation for the probability density,

$$
\frac{\partial p}{\partial t}=-\frac{\partial J}{\partial x}=-\frac{\partial}{\partial x}(v p),
$$

and the corresponding equation for the probability current,

$$
J(x, t)=a p-\frac{1}{2} \frac{\partial}{\partial x}\left(b^{2} p\right)=h p-\frac{b^{2}}{2} \frac{\partial p}{\partial x},
$$

or the current velocity,

$$
v(x, t)=\frac{J}{p}=a-b \frac{\partial b}{\partial x}-\frac{b^{2}}{2} \frac{\partial \ln p}{\partial x},
$$

which with the kinetic prescription becomes

$$
v(x, t)=h-\frac{b^{2}}{2} \frac{\partial \ln p}{\partial x} .
$$

It is also interesting to rewrite the previous equations in the form of a local evolution equation for the entropy. Following $[9,12]$, we define a local entropy density per unit number of trajectories, $s=-\ln p$ (as opposed to an entropy per unit volume which would be $-p \ln p$ ). Since the ensemble of trajectories at a point behaves as a fluid with velocity $v$ [see Eq. (9)], the comoving local entropy balance is given by the material derivative of $s$ [12]:

$$
\frac{d s}{d t} \equiv \frac{\partial s}{\partial t}-v \frac{\partial s}{\partial x}=-\frac{\partial \ln p}{\partial t}-v \frac{\partial \ln p}{\partial x}=\frac{\partial v}{\partial x} .
$$

Using the definition of the current velocity Eq. (12) to express the derivative in the convective term, Eq. (13) becomes

$$
\frac{d s}{d t}=-\frac{1}{p} \frac{\partial p}{\partial t}-\frac{2 v h}{b^{2}}+\frac{2 v^{2}}{b^{2}} .
$$


Thus the infinitesimal "fluid element" moving with velocity $v$ has entropy balance

$$
\frac{d s}{d t}=\frac{d s_{e}}{d t}+\sigma
$$

where $\frac{d s_{e}}{d t}=-\frac{1}{p} \frac{\partial p}{\partial t}-\frac{2 v h}{b^{2}}$ is the entropy flux (the first term is due to net influx or outflux of trajectories in $d x$ due to unsteady conditions, while the second can be interpreted as due to the heat flux into the medium [12]) and $\sigma$ is the local entropy production rate. We will focus on the entropy density production rate, following Refs. [9,12],

$$
\sigma=2 \frac{v^{2}}{b^{2}}
$$

where $\sigma \geqslant 0$, in agreement with the second law of thermodynamics. It will be shown later that $\sigma$ is linked to the local time-symmetry properties of the diffusion process, given by the relative entropy between the PDF of forward and backward trajectories $[10,15]$. Moreover, for vanishing noise $\left(b^{2} \rightarrow 0\right)$ but nonzero $v$, the entropy density production becomes infinite because in that case the backward and forward trajectories become perfectly distinguishable.

\section{B. Steady state}

With natural boundaries, the equilibrium steady-state PDF of the process is given by the potential solution (i.e., probability current identically zero)

$$
p_{\mathrm{E}}(x)=\frac{N}{b^{2}(x)} \exp \left[2 \int^{x} \frac{a\left(x^{\prime}\right)}{b^{2}\left(x^{\prime}\right)} d x^{\prime}\right]=N \exp [-\Phi(x)],
$$

where the effective potential is

$$
\Phi(x)=-2 \int^{x} \frac{h\left(x^{\prime}\right)}{b^{2}\left(x^{\prime}\right)} d x^{\prime},
$$

and $N$ is a normalizing constant (as noted in Ref. [34], the kinetic prescription yields the steady-state PDF in a purely exponential form). Moreover, at steady state Eqs. (7) and (17) give

$$
-\partial_{x} \ln p_{\mathrm{E}}=\partial_{x} s_{\mathrm{E}}=-2\left[a / b^{2}-\partial_{x} \ln b\right]=-2 h / b^{2},
$$

so that, using Eq. (11),

$$
v=\frac{b^{2}}{2} \partial_{x}\left(s-s_{\mathrm{E}}\right)=-\frac{b^{2}}{2} \frac{\partial}{\partial x} \ln \frac{p}{p_{\mathrm{E}}} .
$$

It is also possible to have a nonequilibrium steady state (NESS) on an interval $\left(x_{1}, x_{2}\right)$, involving a constant nonzero current $J^{*}[2,3]$. In general, this gives

$$
p_{\mathrm{NESS}}(x)=N^{\prime} \exp [-\Phi(x)]\left[1-\frac{J^{*}}{N^{\prime}} \int^{x} \frac{2 e^{\Phi\left(x^{\prime}\right)}}{b^{2}\left(x^{\prime}\right)} d x^{\prime}\right],
$$

where formally a NESS potential can be introduced as

$$
p_{\mathrm{NESS}}(x)=N^{\prime \prime} \exp [-\Psi(x)] .
$$

Note that the range of admissible values of the current $J^{*}$ may be restricted by the requirement that the PDF is nonnegative. Within these limits, the current can be altered by adding or removing trajectories at the boundaries. A special case corresponds to the periodic conditions or diffusion on a circle [2,39-41].

For NESS, one can write the previous equation as

$$
v_{\mathrm{NESS}}\left(x, J^{*}\right)=h-\frac{b^{2}}{2} \frac{\partial \ln p_{\mathrm{NESS}}}{\partial x},
$$

where $p_{\text {NESS }}$ is linked to the current $J^{*}$ by Eq. (21). Solving the previous equation allows us to express the NESS potential as

$$
\Psi(x)=-2 \int^{x} \frac{h\left(x^{\prime}\right)-v_{\mathrm{NESS}}\left(x^{\prime}, J^{*}\right)}{b^{2}\left(x^{\prime}\right)} d x^{\prime},
$$

which tends to the effective potential when reducing the value of $J^{*}$, and therefore of $v_{\mathrm{NESS}}$, to zero. The previous equation can also be written as

$$
\Psi(x)=\Phi(x)-\Xi(x),
$$

where $\Xi(x)=-2 \int^{x} \frac{v_{\mathrm{NESS}}\left(x^{\prime}, J^{*}\right)}{b^{2}\left(x^{\prime}\right)} d x^{\prime}$.

In equilibrium steady state $\left(J^{*}=0\right)$, zero entropy production rate, zero current velocity, and reversibility of trajectories are equivalent properties, and all the terms of the entropy balance go to zero. As a result, steady states of 1D free diffusion with natural boundaries are always time-reversible, equilibrium states. For NESS $\left[J^{*} \neq 0, x \in\left(x_{1}, x_{2}\right)\right]$ instead, the material derivative of $s$ is nonzero, and the entropy balance Eq. (14) implies

$$
\frac{d s}{d t}=\frac{\partial v_{\mathrm{NESS}}}{\partial x}=\frac{2 v_{\mathrm{NESS}}}{b^{2}}\left(v_{\mathrm{NESS}}-h\right) .
$$

\section{REVERSED DIFFUSION}

Several aspects of reversed diffusion equations have been previously discussed in the literature $[16,31,42,43]$. Here we aim at defining local kinematic properties of reversed diffusion and their link to the local entropy balance. One way to approach this goal is to note that one can write down an evolution equation in the form of Fokker-Planck equation for the probability density of the state of the system, but with respect to the reversed time $\tau=T-t$, by simply changing the sign of the time derivative in the usual FP Eq. (2) and readjusting the terms as needed, i.e.,

$$
\frac{\partial \hat{p}(x, \tau)}{\partial \tau}=-\frac{\partial}{\partial x}[\hat{a} \hat{p}(x, \tau)]+\frac{1}{2} \frac{\partial^{2}}{\partial x^{2}}\left[\hat{b}^{2} \hat{p}(x, \tau)\right],
$$

where if we choose the diffusion coefficient in reversed time to match the forward diffusion coefficient, $\hat{b}^{2}=b^{2}$, then we choose the drift coefficient in reversed time as

$$
\hat{a}=-a+\frac{1}{p(x, T-\tau)} \frac{\partial}{\partial x}\left[b^{2} p(x, T-\tau)\right] .
$$

Anderson [16] derived equations equivalent to Eqs. (27) and (28) for the two-point joint PDF using the forward and the backward diffusion equations. Equation (27) must have the final solution of the forward process $p(x, T)$ as initial condition $\hat{p}(x, \tau=0)$, while its drift term Eq. (28), containing the drift and the solution of the forward Eq. (2), "guides" the solution of the reversed problem back to the original initial condition $\hat{p}(x, \tau=T)=p(x, 0)$. Thus Eq. (27) is not of predictive value but will merely be used to describe the statistics of time-reversed trajectories. For now, we simply 
observe that by the construction and uniqueness of solutions to a parabolic equation of the form of Eq. (27) with the specified initial condition $\hat{p}(x, \tau=0)=p(x, T)$, we must have $\hat{p}(x, \tau)=p(x, t=T-\tau)$. Other choices of $\hat{p}(x, \tau=0)$ could also be used with the reversed FP Eq. (27) for the purpose of reconstructing statistical subensembles (including diffusion bridges) of the free diffusion trajectories Eq. (1) with initial positions distributed according to $p(x, 0)$, but we will not pursue this here.

Before assigning a meaning to the coefficients in Eq. (27), we must first address the fact that one could construct a reversed-time FP equation of the same form as Eq. (27) for arbitrary choices of reversed-time diffusion coefficient $\frac{1}{2} \hat{b}^{2}$ by choosing a corresponding drift coefficient:

$$
\hat{a}=-a+\frac{1}{2 p(x, T-\tau)} \frac{\partial}{\partial x}\left[\left(\hat{b}^{2}+b^{2}\right) p(x, T-\tau)\right] .
$$

These equations with $\hat{b} \neq b$, however, are nothing but a pointless reformulation of partial differential equation, whereas the choice Eq. (28) corresponding to $\hat{b}=b$ does describe actual statistics of reversed time trajectories. First of all, the solution trajectories of a stochastic differential equation or any Markov process can be shown directly by the definition to be Markov processes also in reversed time. Second, the reversed time trajectories will have the same qualitative local character and smoothness as the forward time trajectories, so we infer that the infinitesimal generator of the time-reversed trajectories should again be a second-order differential operator corresponding to a diffusion process with some drift coefficient $\hat{\tilde{a}}$ and diffusion coefficient $\frac{1}{2} \hat{\tilde{b}}^{2}$. Now, the diffusion coefficient must be the same in both time directions $(\hat{\tilde{b}}=b)$, as it characterizes the roughness of the paths, so that the size of independent increments cannot depend on the direction in which they are traversed. This same idea is behind the absolute continuity of probability measures of solution paths to SDEs with respect to each other if and only if their diffusion coefficients agree (e.g., Ref. [44], Ch. 7). The drift coefficient can, and generally will, suffer a bias and be different in reversed time due to the steering needed to bring the trajectories to the appropriate final probability distribution (the initial condition for the forward equation). We can see that the appropriate drift coefficient is the formula of Eq. (28) obtained above as follows: because the time-reversed trajectories are again a diffusion process, the probability density for the time-reversed trajectories must satisfy a Fokker-Planck equation with the correct drift and diffusion coefficients

$$
\frac{\partial \hat{p}(x, \tau)}{\partial \tau}=-\frac{\partial}{\partial x}[\hat{\tilde{a}} \hat{p}(x, \tau)]+\frac{1}{2} \frac{\partial^{2}}{\partial x^{2}}\left[\hat{\tilde{b}}^{2} \hat{p}(x, \tau)\right] ;
$$

agreement between Eqs. (27) and (30), together with the observation that we must have $b=\hat{\tilde{b}}$, forces

$$
-\frac{\partial}{\partial x}[\hat{\tilde{a}} \hat{p}(x, \tau)]=-\frac{\partial}{\partial x}[\hat{a} \hat{p}(x, \tau)] .
$$

This in turn implies that $(\hat{\tilde{a}}-\hat{a}) \hat{p}(x, \tau)$ is a constant, which must be zero under the reasonable assumption that $\hat{p}$ decays for large $|x|$ faster than $\hat{\tilde{a}}$ and $\hat{a}$ grow. This then implies that $\hat{\tilde{a}}=\hat{a}$, meaning that Eq. (28) is indeed the drift coefficient in the diffusion process describing the time-reversed trajectories.
We can therefore write a SDE (in the Itô form) for the timereversed trajectories, with time-dependent drift, corresponding to Eq. (27)

$$
d \hat{X}=\hat{a}(\hat{X}, \tau) d \tau+b(\hat{X}) \widehat{d W}(\tau),
$$

or using the kinetic prescription

$$
d \hat{X}=\hat{h}(\hat{X}, \tau) d \tau+b(\hat{X}) \star \widehat{d W}(\tau),
$$

where

$$
\begin{aligned}
\hat{h} & =\hat{a}-b \partial_{x} b=-a+b \partial_{x} b+b^{2} \partial_{x} \ln p \\
& =-h+b^{2} \partial_{x} \ln p=-v+\frac{b^{2}}{2} \partial_{x} \ln p,
\end{aligned}
$$

with the last equality following from Eq. (11). Equation (34) is the generalization to the case of multiplicative noise of the so-called "dual condition" for additive noise [42]. Note that the previous drift can also be written as

$$
\hat{h}=h-2 v \text {. }
$$

As with Eq. (27), the previous SDEs must have their initial positions distributed statistically according to the final PDF of the free forward process, $p(x, T)$. We also used "hats" for the reversed time Wiener process as reminders of the fact that, when different realizations of the Wiener process are used for the forward and reversed processes, only the ensemble of trajectories is reconstructed, without achieving specific strong correspondence among backward and forward trajectories. To have a strong correspondence between trajectories, the same realization that generated the forward process must be used also in the reversed time $\tau$; in such a case, however, the increments of the time-reversed Wiener process are not independent of the "previous" (in $\tau$ ) values of $\hat{X}$, but rather of the "future" ones (see also Ref. [16]).

It is also instructive to consider the stochastic interpretation of the time-reversed drift and diffusion. Similarly to the interpretation of Eqs. (1) and (2) in the forward time $t$, it is possible to have causal or forward (in $\tau$ ) interpretation of the increments of $\hat{X}$ of the stochastic process for Eqs. (27) and (32). Thus, with

$$
\Delta \hat{X}=\left.(\hat{X}(\tau+\Delta \tau)-\hat{X}(\tau))\right|_{\hat{X}(\tau)=x},
$$

it follows that $\langle\Delta \hat{X}\rangle=\hat{a} \Delta \tau$ and $\left\langle(\Delta \hat{X})^{2}\right\rangle=\hat{b}^{2}(\hat{X}) \Delta \tau$ for $\Delta \tau \rightarrow 0$. In other words, for $\Delta \tau \rightarrow 0, \Delta \hat{X} / \Delta \tau$ is Gaussian with mean $\hat{a}$ and variance $b^{2} / \Delta \tau$.

The reversed-diffusion drift $\hat{a}$ can be written in a form that separates the contributions due to the evolution of the forward PDF $p(x, T-\tau)$ from the one solely due to the forward drift and diffusion,

$$
\hat{a}=-\left(a-2 b \frac{\partial b}{\partial x}\right)+b^{2} \frac{\partial \ln p(x, T-\tau)}{\partial x} .
$$

Note that the correction $2 b \frac{\partial b}{\partial x}$ is present only in the case of multiplicative noise. The time-reversed drift $\hat{a}$ is also linked to the current velocity,

$$
\hat{a}=a-\frac{2}{p}\left[a p-\frac{1}{2} \frac{\partial}{\partial x}\left(b^{2} p\right)\right]=a-\frac{2 J}{p}=a-2 v,
$$

which is similar in form to Eq. (35). This form of relationship between forward drift, reversed drift, and current velocity is 
valid for any prescription with respect to their specific drifts, including the one of Stratonovich (i.e., if the Stratonovich drift is $g=a-\frac{1}{2} b \partial_{x} b$, then $\hat{g}=g-2 v$ ).

In terms of the hydrodynamic description, the reversed-time diffusion is simply

$$
\frac{\partial \hat{p}}{\partial \tau}=-\frac{\partial}{\partial x}(\hat{v} \hat{p})
$$

where, using the definition of the current velocity Eq. (11) along with Eq. (34),

$$
\hat{v}=-h+\frac{b^{2}}{2} \partial_{x} \ln p=-v .
$$

For steady states with zero current [i.e., corresponding to the potential solution (17)], $v=0$ and, thus, $\hat{a}=a$. Hence, the reversed equation is exactly the same as the forward equation (thus the process is in equilibrium with zero entropy production). This confirms what was previously mentioned regarding the time reversibility (i.e., equilibrium) of all $1 \mathrm{D}$ diffusion steady-states with natural boundary conditions (zero current) [Eq. (16)]. This is not the case for NESS, where $\hat{a}=a-2 v_{\mathrm{NESS}}$.

\section{LOCAL STOCHASTIC VELOCITY AND ACCELERATION}

Because of the nature of the white-noise fluctuations, the trajectories of $X(t)$ are nondifferentiable and cross a given level $x$ an infinite number of times during an infinitesimal time interval [1]. This makes the definition of the local velocity and acceleration possible only when defined with respect to a finite time interval. Consider a finite but small temporal interval $\Delta t$, as shown in Fig. 1, and define, using the centered differences around $X(t)$ :

$$
\dot{X} \mid x=\frac{\left.(X(t+\Delta t / 2)-X(t-\Delta t / 2))\right|_{X(t)=x}}{\Delta t} .
$$

Setting $X(t)=\hat{X}(t)=x$, the numerator in the previous expression can be seen as $(X(t+\Delta t / 2)-X(t))-(X(t-$ $\Delta t / 2)-X(t))=(X(t+\Delta t / 2)-x)-(\hat{X}(\tau+\Delta t / 2)-x)=$ $\Delta X_{\Delta t / 2}-\Delta \hat{X}_{\Delta t / 2}$. Because of the Markov property for both backward and forward processes, these two terms are independent. Moreover, based on Eqs. (4) and (36), we have that $\Delta X_{\Delta t / 2} \sim \mathcal{N}\left[a \Delta t / 2, b^{2} \Delta t / 2\right]$ and $\Delta \hat{X}_{\Delta t / 2} \sim \mathcal{N}\left[\hat{a} \Delta t / 2, b^{2} \Delta t / 2\right]$, where $\mathcal{N}$ stands for normal

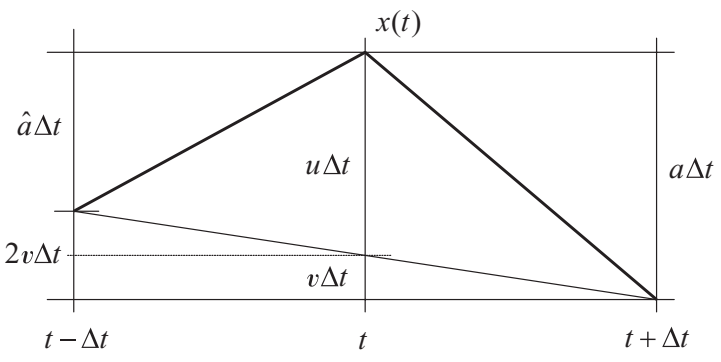

FIG. 1. Sketch of the geometric meaning of the various mean velocities. distributions with mean and variance given by its respective arguments. Their difference thus gives

$$
\dot{X} \mid x \sim \mathcal{N}\left[v, \frac{b^{2}}{\Delta t}\right],
$$

where we have used the fact that $a-\hat{a}=2 v$, which follows from Eq. (38). Based on this, the local current velocity from Eq. (11), $v=\langle\dot{X} \mid x\rangle$, can also be interpreted as half the difference between the forward and backward drift:

$$
v=\frac{a-\hat{a}}{2} \text {. }
$$

We can also define a local acceleration

$$
\ddot{X} \mid x=\frac{\Delta^{2} X}{\Delta t^{2}},
$$

where

$$
\begin{aligned}
\Delta^{2} X & =\left.(X(t+\Delta t)-2 X(t)+X(t-\Delta t))\right|_{X(t)=x} \\
& =\Delta X+\Delta \hat{X} .
\end{aligned}
$$

It thus follows that

$$
\ddot{X} \mid x \sim \mathcal{N}\left[\frac{a+\hat{a}}{\Delta t}, \frac{2 b^{2}}{(\Delta t)^{3}}\right]=\mathcal{N}\left[\frac{2(a-v)}{\Delta t}, \frac{2 b^{2}}{(\Delta t)^{3}}\right] .
$$

One can also introduce a fluctuation velocity defined as the ensemble average of the arithmetic mean of forward and backward instantaneous velocities,

$$
u=\frac{1}{2}\left\langle\lim _{\Delta t \rightarrow 0} \frac{\Delta X+\Delta \hat{X}}{\Delta t}\right\rangle=\frac{a+\hat{a}}{2},
$$

where the averaging allows the actual passage to the limit. The fluctuation velocity $u$ is directly related to the mean local acceleration as

$$
\langle\ddot{X} \mid x, t\rangle=(a+\hat{a}) / \Delta t=2 u / \Delta t .
$$

Since the process is nondifferentiable, the mean local acceleration does not converge to a finite limit as $\Delta t \rightarrow 0$.

It should be noted that Eq. (3) only includes leading-order terms corresponding to a forward Euler discretization of a differential equation. Higher order terms are neglected, the most important of which is a non-Gaussian random term with mean zero and variance proportional to $(\Delta t)^{2}$, of the form

$$
b[X(t)] b^{\prime}[X(t)] \int_{t}^{t+\Delta t}\left[W\left(t^{\prime}\right)-W(t)\right] d W\left(t^{\prime}\right),
$$

and uncorrelated with the $b[X(t)] \Delta W(t)$ term in Eq. (3), plus a standard second-order deterministic error term $a[X(t)] a^{\prime}[X(t)](\Delta t)^{2}$ (see, e.g., Ref. [45], Sec. 5.5). Thus, the stochastic velocity $\dot{X} \mid x$ is more precisely a sum of the large Gaussian random variable written above plus a non-Gaussian random variable with $O(\Delta t)$ mean and $O(1)$ variance. Similarly, the stochastic acceleration $\ddot{X} \mid x$ is a sum of the large Gaussian random variable written above, plus a non-Gaussian random variable with $O(1)$ mean and $O(1 / \Delta t)$ variance. Such corrections are not small, but they are not as large as the Gaussian random components explicitly described. While under some circumstances they could be important, when the manipulations of these random variables 
is essentially multiplicative they do not play a leading order role, and therefore do not affect the previous conclusions.

\section{A. Kramers equation (steady state)}

We now consider the particular case of a diffusion process corresponding to Langevin particle dynamics. First we consider the Kramers equation as a foundational model based on Newton's law (e.g., Ref. [2], p. 155)

$$
\left\{\begin{array}{l}
\dot{X}=V \\
m d V=F(X) d t-\beta V d t+\sqrt{2 k_{B} \beta T} d W(t) .
\end{array}\right.
$$

Here $m$ is mass, $V$ is the velocity variable, $F(X)$ is the applied force, $\beta$ is a friction constant, $T$ is the absolute temperature (assumed to be constant in this subsection for simplicity), and $k_{B}$ is Boltzmann's constant. Because the thermal noise appears here as a force in the velocity equation, the meansquare velocity and acceleration are well-defined and can be shown to satisfy (see, e.g., Ref. [46])

$$
\left\langle\dot{X}^{2} \mid x, t\right\rangle_{\mathrm{Kr}}=\frac{k_{B} T}{m} \quad \text { and } \quad\langle\ddot{X} \mid x, t\rangle_{\mathrm{Kr}}=\frac{F(x)}{m} .
$$

In the Smoluchovski approximation, corresponding to friction dominating inertia $(\gamma=\beta / m \rightarrow \infty$; e.g., Ref. [2], p. 197), we obtain a reduced equation,

$$
d X=\frac{F(X)}{\beta}+\sqrt{\frac{2 k_{B} T}{\beta}} d W(t),
$$

corresponding to a diffusion Eq. (1) with coefficients

$$
a=\frac{F(X)}{\beta} \quad \text { and } \quad b^{2}=\frac{2 k_{B} T}{\beta} .
$$

From these expressions, and using the results of the previous section, Eqs. (46) and (42), for steady state (as previously noticed, $\hat{a}=a$ in steady state),

$$
\langle\ddot{X} \mid x, t\rangle_{\mathrm{Sm}}=\frac{2 a}{\Delta t} \quad \text { and } \quad\left\langle\dot{X}^{2} \mid x, t\right\rangle_{\mathrm{Sm}}=\frac{b^{2}}{\Delta t} .
$$

In terms of the $a$ and $b$ coefficients, we can write the corresponding statistics for the full Kramers equation as

$$
\langle\ddot{X} \mid x, t\rangle_{\mathrm{Kr}}=a \gamma \quad \text { and } \quad\left\langle\dot{X}^{2} \mid x, t\right\rangle_{\mathrm{Kr}}=\frac{b^{2}}{2} \gamma .
$$

Now these statistics need not agree between the two models, because the Kramers statistics are obtained from a finite value of $\gamma$ with $\Delta t \rightarrow 0$, whereas the Smoluchowski statistics are obtained from a finite value of $\Delta t$ with $\gamma \rightarrow \infty$. Nonetheless, the statistics do agree in a sort of intermediate regime, where $\gamma=\beta / m=2 / \Delta t$ is taken small, meaning that the friction parameter in the Kramers equation is balanced with the time interval used to compute statistics in the Smoluchowski equation. This can be understood as either $\Delta t$ or $2 / \gamma$ serving as a smoothing time scale (either through sampling or dynamics) in evaluating the velocity and acceleration statistics.

\section{B. Canonical form of the transient solution and link with the Pope-Ching formula}

Equations (2) and (27) can be linked to Nelson's stochastic dynamics [26,27], as well as be used to introduce a generalized wave equation. These considerations in turn allow a geometric interpretation of the various mean velocities introduced before. We begin by rewriting both equations at the same instant $t$ and making the time derivative explicit. We also use the fact that $\hat{p}(\tau=T-t)=p(t), \hat{p}(\tau+\Delta \tau=T-t-\Delta t)=$ $p(t-\Delta t)$, and that the time increment $\Delta \tau$ is equal to $\Delta t$. With this in mind, Eqs. (2) and (27) become, respectively,

$$
\begin{aligned}
\frac{p(x, t+\Delta t)-p(x, t)}{\Delta t} & =-\frac{\partial}{\partial x}(a p)+\frac{1}{2} \frac{\partial^{2}}{\partial x^{2}}\left(b^{2} p\right), \\
\frac{p(x, t-\Delta t)-p(x, t)}{\Delta t} & =-\frac{\partial}{\partial x}(\hat{a} p)+\frac{1}{2} \frac{\partial^{2}}{\partial x^{2}}\left(\hat{b}^{2} p\right),
\end{aligned}
$$

with small $\Delta t$. As already noticed by Nelson [27], taking the difference of the previous equations and dividing by two immediately yields the hydrodynamic Eq. (9). The sum of Eqs. (56) and (57) instead yields

$$
\Delta t \frac{\Delta^{2} p}{\Delta t^{2}}=-\frac{\partial}{\partial x}(2 u p)+\frac{\partial^{2}}{\partial x^{2}}\left(b^{2} p\right),
$$

after having noticed that the sum of the drifts provides the fluctuation velocity $u$, while for the diffusion terms (from Sec. III) one has $\left(b^{2}+\hat{b}^{2}\right) / 2=b^{2}$. Using Eqs. (42) and (48), and dividing by $\Delta t$, one has formally a generalized wave equation as in Refs. [32,48]:

$$
\frac{\partial^{2} p}{\partial t^{2}}=-\frac{\partial}{\partial x}(\langle\ddot{X} \mid x, t\rangle p)+\frac{\partial^{2}}{\partial x^{2}}\left(\left\langle\dot{X}^{2} \mid x\right\rangle p\right) .
$$

However, because of the diffusive limit, this is only formally a wave equation, since the coefficients on the right-hand side blow up as $\Delta t \downarrow 0$. In this limit, Eq. (58) is actually

$$
0=-\frac{\partial}{\partial x}(2 u p)+\frac{\partial^{2}}{\partial x^{2}}\left(b^{2} p\right),
$$

which can be solved, considering that the first integration constant is always zero because the externally imposed currents (if any) in Eqs. (56) and (57) cancel out. The solution,

$$
\begin{aligned}
p(x, t) & =\frac{N(t)}{b(x)^{2}} \exp \left[2 \int^{x} \frac{u\left(x^{\prime}, t\right)}{b\left(x^{\prime}\right)^{2}} d x^{\prime}\right] \\
& =N(t) \exp \left[2 \int^{x} \frac{h\left(x^{\prime}\right)-v\left(x^{\prime}, t\right)}{b\left(x^{\prime}\right)^{2}} d x^{\prime}\right],
\end{aligned}
$$

provides an exponential form of the transient PDF, in agreement with the fact that a canonical distribution should exist in the diffusion approximation (which physically corresponds to a local and quasistatic equilibrium). Note that Eq. (61) could have also been obtained directly from Eq. (28).

In steady state, the fluctuation velocity $u=a-v_{\text {NESS }}$ and the PDF in Eq. (21) can also be written as

$$
p_{\mathrm{NESS}}(x)=N \exp \left(2 \int^{x} \frac{h-v_{\mathrm{NESS}}}{b^{2}} d x^{\prime}\right),
$$

in agreement with Eq. (24). In terms of local velocity and acceleration,

$$
p_{\mathrm{NESS}}(x)=\frac{N}{\left\langle\dot{X}^{2} \mid x\right\rangle} \exp \int^{x} \frac{\langle\ddot{X} \mid y\rangle}{\left\langle\dot{X}^{2} \mid y\right\rangle} \mathrm{d} y,
$$

where the expression in the exponent is well-defined in the $\Delta t \downarrow 0$ limit, whereas in the prefactor the normalization constant $N$ would scale in proportion to $1 / \Delta t$. Equation (63) 
was previously obtained by Pope and Ching with reference to general twice-differentiable stationary signals [32,47,48]. Note that $\langle\ddot{X} \mid x\rangle$ depends on $J^{*}$ for NESS. With zero current velocity, Eq. (38) gives $\hat{a}=a$, and thus $\langle\ddot{X} \mid x\rangle=2 a / \Delta t$, which in turn yields the equilibrium solution Eq. (17).

\section{Geometric interpretation of fluctuation velocity}

Several local mean velocities have been introduced in the course of the previous sections: the drift $a$, defined after Eq. (3), the current velocity $v$ [see Eq. (11)], the reversed drift $\hat{a}$ [Eqs. (28) and (37) and (38)], and the fluctuation velocity $u$ [Eq. (47)],

$$
u=\frac{a+\hat{a}}{2}=a-v=\frac{1}{2 p} \frac{\partial}{\partial x}\left(b^{2} p\right),
$$

where the last equality is due to Eq. (37). At equilibrium steady state (e.g., $v=0$ ), the fluctuation velocity $u$ is equal to the drift $a$. Combining Eqs. (64), (41), and (47) makes the geometric meaning of this velocity more clear, showing that $u$ is the average rate of vertical displacement from the straight line connecting the end points $\langle X(t+\Delta t)\rangle,\langle X(t-$ $\Delta t)\rangle$ of trajectories passing through $x$ at time $t$. This geometric interpretation is shown in Fig. 1.

By considering Eq. (11), the fluctuation velocity can also be split into two parts,

$$
u=u_{\mathrm{O}}+u_{\mathrm{T}},
$$

where the first component is related to the change in trajectory concentration (thus related to an osmotic potential), and for this reason called osmotic velocity by Nelson [27],

$$
u_{\mathrm{O}}=\frac{b^{2}}{2} \frac{\partial \ln p(x, t)}{\partial x},
$$

while the other component is related to the drift due to the multiplicative noise. This may be termed thermal velocity because of its relation to thermophoresis [36,49-52]) and it is linked to the gradient of the non-uniform noise intensity or local temperature $b^{2}$ (see Sec. III),

$$
u_{\mathrm{T}}=\frac{1}{2} \frac{\partial b^{2}}{\partial x}=b b^{\prime}
$$

The previous observations also allow the decomposition of current velocity into its three components,

$$
v=a-\left(u_{\mathrm{O}}+u_{\mathrm{T}}\right),
$$

implying that at any instant there is a momentum (per unit mass) balance among these different components (note also that $h=a-u_{\mathrm{T}}$ ). When each term of the previous equation is multiplied by $p$, one obtains the respective components of the probability current of Landauer [Ref. [36], Eq. (4.1)].

At equilibrium $(v=0)$ the drift velocity balances the sum of osmotic and thermal velocities, which is another way of stating the fluctuation-dissipation relation or generalized Einstein relation,

$$
a=u_{\mathrm{O}, \mathrm{E}}+u_{\mathrm{T}}=\frac{b^{2}}{2} \frac{\partial \ln p_{\mathrm{E}}(x)}{\partial x}+\frac{1}{2} \frac{\partial b^{2}}{\partial x},
$$

or with the kinetic prescription Eq. (7),

$$
h=\frac{b^{2}}{2} \frac{\partial \ln p_{\mathrm{E}}(x)}{\partial x} .
$$

In NESS instead [cf. Eq. (23)],

$$
u_{\mathrm{O}, \mathrm{NESS}}=\frac{b^{2}}{2} \frac{\partial \ln p_{\mathrm{NESS}}(x)}{\partial x}=h-v_{\mathrm{NESS}}=\frac{b^{2}}{2} \frac{\partial \Psi}{\partial x} \text {. }
$$

\section{ENTROPY PRODUCTION}

It was shown in Sec. III that when the current velocity $v$ is different from zero, the system is out of equilibrium with positive entropy production rate $\sigma$. Both the current velocity Eq. (11) and the entropy production rate Eq. (16) are mean quantities. In Sec. IV we discussed the statistical properties of the random variable underlying the current velocity. In a similar manner, we can now discuss the properties of the random variable associated with the entropy production rate. This is especially important, since the random fluctuations around the mean entropy production rate have been the subject of intense study in the context of the fluctuation theorem in nonequilibrium statistical mechanics [10,11,14].

Recent developments (e.g., Refs. [10,14]) have shown that the local entropy production rate can be expressed as the relative entropy between forward and backward velocity distributions, $p_{\dot{X}}(\dot{x} \mid x, t)$ and $p_{\dot{X}}(\dot{x} \mid x, t)$, conditioned on the current state $x$ and time $t[14,15]$. Noting that $p_{\dot{\hat{X}}}(\dot{x} \mid x, t)=$ $p_{\dot{X}}(-\dot{x} \mid x, t)$,

$$
\sigma(x, t)=\frac{1}{\Delta t} \int d \dot{x} p_{\dot{X}}(\dot{x} \mid x, t) \ln \frac{p_{\dot{X}}(\dot{x} \mid x, t)}{p_{\dot{X}}(-\dot{x} \mid x, t)}
$$

gives a trajectory-based or "microscopic/local" interpretation of the mean entropy production rate. The explicit $1 / \Delta t$ factor is needed to obtain a nontrivial $\Delta t \rightarrow 0$ limit of the expression on the right-hand side; the PDF for the particle velocity $\dot{X}$ is understood here to be regularized with respect to a small but finite time interval $\Delta t$ as in Sec. IV. Equation (72) complies with the intuitive interpretation of time reversibility, in that a process is time-symmetric only if the same distributions of slopes are found going either forward or backward in time. Therefore, in relation to the diffusion process with locally Gaussian increments of interest here, Eq. (72) also provides a local interpretation of Eq. (16). It can be shown that the relative entropy between two Gaussian distributions with the same variance $b^{2} / \Delta t$ and opposite means $(v$ and $-v)$ is equal to twice the square of the mean $\left(v^{2}\right)$ divided by the variance $\left(b^{2} / \Delta t\right)$, which inserted in Eq. (72) gives Eq. (16).

We can also show that the PDF $p_{Z}(z)$ of the random variable whose average gives the entropy production rate,

$$
Z(x, t)=\frac{1}{\Delta t} \ln \frac{p_{\dot{X}}(\dot{x} \mid x, t)}{p_{\dot{X}}(-\dot{x} \mid x, t)},
$$

satisfies the so-called fluctuation theorem [15,53-55],

$$
p_{\Delta Y}(-y)=p_{\Delta Y}(y) e^{-y},
$$

where $\Delta Y=Z \Delta t$ is the random fluctuation during a regularized time step $\Delta t$ whose average gives the entropy produced during that time step. While this is valid in great generality 
[15], we can derive it simply in our case using the Gaussian distribution for $\dot{X} \mid x$ [Eq. (42)] to write

$$
Z(x, t)=\frac{2 v(x, t)}{b^{2}(x, t)} \dot{X} \mid x,
$$

which can be seen to be itself approximately Gaussian,

$$
Z \sim \mathcal{N}\left(\sigma, \frac{2 \sigma}{\Delta t}\right)
$$

Equation (74) now follows by explicit manipulation of the Gaussian distribution

$$
\Delta Y \sim \mathcal{N}(\sigma \Delta t, 2 \sigma \Delta t) .
$$

The random variable $\exp \left(\int_{0}^{t} Z\left(X\left(t^{\prime}\right), t^{\prime}\right) \mathrm{d} t^{\prime}\right)$ is also related to the Radon-Nykodim derivative between Eq. (1) and its reverse, Eq. (32), through the Girsanov theorem (e.g., Ref. [5], p. 164). The latter relates the probabilistic properties of two diffusion processes taking place on a common interval $[0, t]$. In the present case, the two processes are the free forward diffusion

$$
d X=a d t+b d W
$$

and a time-reversed one, with the same diffusion term [see Eq. (38)]:

$$
d \hat{X}=\hat{a} d \tau+b \widehat{d W}(\tau)=a d \tau-2 v d \tau+b \widehat{d W}(\tau),
$$

both with the same (possibly random) initial conditions. According to the Girsanov theorem, the ratio of their path measures on $[0, t]$ (i.e., their Radon-Nykodim derivative) is

$$
\begin{aligned}
\frac{\mathrm{d} P_{X}}{\mathrm{~d} P_{\hat{X}}}= & \exp \left[\int_{0}^{t} \frac{2 v\left(X_{t^{\prime}}, t^{\prime}\right)}{b^{2}\left(X_{t^{\prime}}, t^{\prime}\right)} \mathrm{d} X_{t^{\prime}}\right] \\
& \times \exp \left\{\int_{0}^{t} \frac{2 v\left(X_{t^{\prime}}, t^{\prime}\right)\left[v\left(X_{t^{\prime}}, t^{\prime}\right)-a\left(X_{t^{\prime}}, t^{\prime}\right)\right]}{b^{2}\left(X_{t^{\prime}}, t^{\prime}\right)} \mathrm{d} t^{\prime}\right\} \\
= & \exp \left\{\int_{0}^{t} \frac{2 v\left(X_{t^{\prime}}, t^{\prime}\right)}{b\left(X_{t^{\prime}}, t^{\prime}\right)} \mathrm{d} W_{t^{\prime}}+\frac{1}{2} \int_{0}^{t}\left[\frac{2 v\left(X_{t^{\prime}}, t^{\prime}\right)}{b\left(X_{t^{\prime}}, t^{\prime}\right)}\right]^{2} \mathrm{~d} t^{\prime}\right\} .
\end{aligned}
$$

The above expression is evaluated at a random path $X_{t}$ [referring for short here to $X(t)]$ and so is also a random variable. This Radon-Nikodym derivative can also be expressed, in a NESS, in terms of a single Stratonovich integral [5], as in Eq. (3.64) of Ref. [8],

$$
\frac{\mathrm{d} P_{X}}{\mathrm{~d} P_{\hat{X}}}=\exp \left[\int_{0}^{t} \frac{2 v\left(X_{t^{\prime}}, t^{\prime}\right)}{b^{2}\left(X_{t^{\prime}}, t^{\prime}\right)} \circ \mathrm{d} X_{t^{\prime}}\right],
$$

as can be shown through using the transformation rule between Stratonovich and Itô integrals (now suppressing all arguments indicating evaluation along the random paths $X_{t^{\prime}}$ ),

$$
\begin{aligned}
\int_{0}^{t} \frac{2 v}{b^{2}} \circ \mathrm{d} X_{t^{\prime}}= & \int_{0}^{t} \frac{2 v}{b^{2}} \mathrm{~d} X_{t^{\prime}}+\frac{1}{2} \int_{0}^{t} \frac{\partial}{\partial x}\left[\frac{2 v}{b^{2}}\right] \mathrm{d}[X, X]\left(t^{\prime}\right) \\
= & \int_{0}^{t} \frac{2 v}{b^{2}} a \mathrm{~d} t^{\prime}+\int_{0}^{t} \frac{2 v}{b^{2}} b \mathrm{~d} W_{t^{\prime}} \\
& +\frac{1}{2} \int_{0}^{t} \frac{\partial}{\partial x}\left[\frac{2 v}{b^{2}}\right] \mathrm{d}[X, X]\left(t^{\prime}\right),
\end{aligned}
$$

and then using Eqs. (11) and (13) to write the correction term involving the quadratic variation of the diffusion process $\mathrm{d}[X, X]\left(t^{\prime}\right)=b^{2}\left(X_{t^{\prime}}, t^{\prime}\right)$ as

$$
\begin{aligned}
\frac{1}{2} & \int_{0}^{t} \frac{\partial}{\partial x}\left[\frac{2 v}{b^{2}}\right] \mathrm{d}[X, X]\left(t^{\prime}\right)=\int_{0}^{t}\left[\frac{\frac{\partial v}{\partial x}}{b^{2}}-2 \frac{v \frac{\partial b}{\partial x}}{b^{3}}\right] b^{2} \mathrm{~d} t^{\prime} \\
& =\int_{0}^{t}\left[-\frac{\partial \ln p}{\partial t^{\prime}}-v \frac{\partial \ln p}{\partial x}-2 \frac{v(a-v)}{b^{2}}+v \frac{\partial \ln p}{\partial x}\right] \mathrm{d} t^{\prime} \\
& =\int_{0}^{t} 2 \frac{v(v-a)}{b^{2}} \mathrm{~d} t^{\prime},
\end{aligned}
$$

where the partial time derivative vanishes because we are here considering NESS. We stress that Eq. (80) applies for evolving probability distributions, while the Stratonovich integral Eq. (81) only applies for NESS.

If we discretize the time integrals in Eq. (80) with the same time step $\Delta t$ as in our regularization for local velocities and accelerations, then upon comparison with Eq. (76), we note that each summand in the discretized integral is, to leading order, statistically equivalent to $Z\left(X_{t^{\prime}}, t^{\prime}\right) \Delta t$ for the corresponding value of $t^{\prime}$. More precisely, the summands in the discretized integrals of Eq. (80) form a Markov process with respect to the filtration generated by the underlying Wiener process $W(t)$, as does the sequence $\left[Z\left(X_{n \Delta t}, n \Delta t\right)\right]_{n=0}^{\lfloor t / \Delta t\rfloor}$, with the same transition density, as can be checked from Eqs. (75) and (41). Consequently, in the $\Delta t \rightarrow 0$, we can write

$$
Y(t)=\ln \frac{d P_{X}}{d P_{\hat{X}}}=\int_{0}^{t} Z\left(X_{t^{\prime}}, t^{\prime}\right) d t^{\prime} .
$$

Finally, taking statistical expectation, and noting that the first term is zero, being a regular Itô integral, one obtains

$$
\begin{aligned}
\left\langle\ln \frac{d P_{X}}{d P_{\hat{X}}}\right\rangle & =\left\langle\frac{1}{2} \int_{0}^{t} \frac{\left(2 v\left(X_{t^{\prime}}\right)\right)^{2}}{b\left(X_{t^{\prime}}\right)^{2}} d t^{\prime}\right\rangle \\
& =\int_{0}^{t} \int_{-\infty}^{\infty} p\left(x, t^{\prime}\right) \sigma\left(x, t^{\prime}\right) d x d t^{\prime},
\end{aligned}
$$

which is the global entropy production over the considered time interval.

The previous considerations also provide an interesting SDE for the entropy production. By taking the logarithm and differentiating Eq. (80), one obtains

$$
d Y=2\left[\frac{v(X)}{b(X)}\right]^{2} d t+\frac{2 v(X)}{b(X)} d W_{t},
$$

which should be coupled to Eq. (1), and where the two equations are driven by the same Wiener noise and are interpreted in the Itô sense. The previous SDE describes the stochastic production of entropy.

A stochastic path representation of entropy production has also been given in Refs. [7,8] (see also Ref. [13]) for $n$-dimensional systems in NESS, using the Girsanov theorem and an action functional related to the ratios of probabilities of forward and backward paths. To make contact with these derivations, it is useful to rewrite the FP equation of free diffusion, making the possible presence of currents at NESS explicit. Using Eq. (71), Eq. (8) becomes

$$
\frac{\partial p}{\partial t}=-\frac{\partial}{\partial x}\left[\left(v_{\mathrm{NESS}}+u_{\mathrm{O}, \mathrm{NESS}}\right) p\right]+\frac{\partial}{\partial x}\left(\frac{b^{2}}{2} \frac{\partial p}{\partial x}\right),
$$


or in terms of an Itô SDE similar to Eq. (3.53) of Ref. [8], recalling Eqs. (7) and (71),

$$
d X=\left[v_{\mathrm{NESS}}-\frac{1}{2} b^{2} \partial_{x} \Psi(x)+\frac{1}{2} \partial_{x} b^{2}\right] d t+b d W .
$$

Note how the first two terms of the drift depend on $J^{*}$, and that the first term tends to zero, while the second one tends to $h$, as $J^{*} \rightarrow 0$. In NESS, the drift of the reversed SDE is $\hat{a}=a-$ $2 v_{\text {NESS }}$, as previously pointed out (see end of Sec. III), so that applying the Girsanov theorem as before, the developments of Ref. [8] applied to Eq. (86) agree with our entropy production rate at NESS.

\section{SIMPLE EXAMPLES}

In the classical example of the Wiener process [2],

$$
d X=\sqrt{D} d W(t),
$$

we have $a=0$ and $b^{2}=D$ constant. As is well known, for deterministic initial condition $X(t=0)=x_{0}$, the cor-
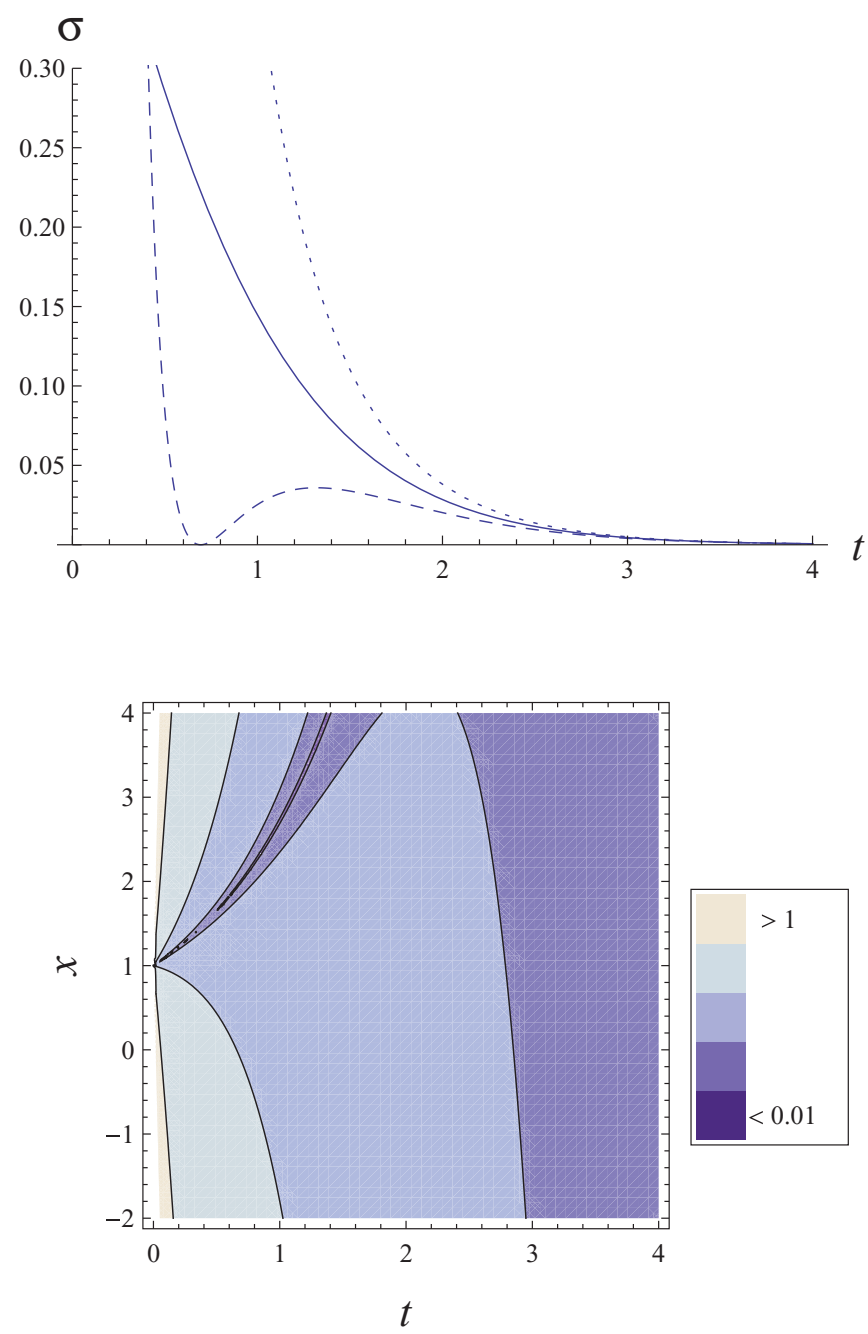

FIG. 2. (Color online) Top panel: Local entropy production $\sigma(x, t)$ for the OU process as a function of time for $x=2$ (dashed), $x=1$ (solid), and $x=0$ (dotted); parameters: $x_{0}=1, D=1, k=1$. Bottom panel: two-dimensional contour plot of $\sigma(x, t)$; parameters: $D=1, k=1$. responding FP equation is simply the heat equation with delta-concentrated initial data, with solution

$$
p(x, t)=\frac{1}{\sqrt{2 \pi D t}} e^{-\frac{\left(x-x_{0}\right)^{2}}{2 D t}} .
$$

Thus, in this case,

$$
v(x, t)=0-\frac{D}{2} \frac{\partial \ln p(x, t)}{\partial x}=\frac{x-x_{0}}{2 t},
$$

which is just half the mean velocity of the particle up to time $t$, and $\sigma=\frac{\left(x-x_{0}\right)^{2}}{2 D t^{2}}$.

The second example is the Ornstein-Uhlenbeck (OU) process, with linear drift and constant diffusion [2]:

$$
d X=-k X d t+\sqrt{D} d W(t) .
$$

For deterministic initial condition $X(t=0)=x_{0}$, the probability density for the solution at time $t$ (see Ref. [2], p. 76) is a Gaussian with mean and variance

$$
\mu_{\mathrm{OU}}=x_{0} e^{-k t} \quad \operatorname{var}_{\mathrm{OU}}=\frac{D}{2 k}\left(1-e^{-2 k t}\right),
$$

respectively.
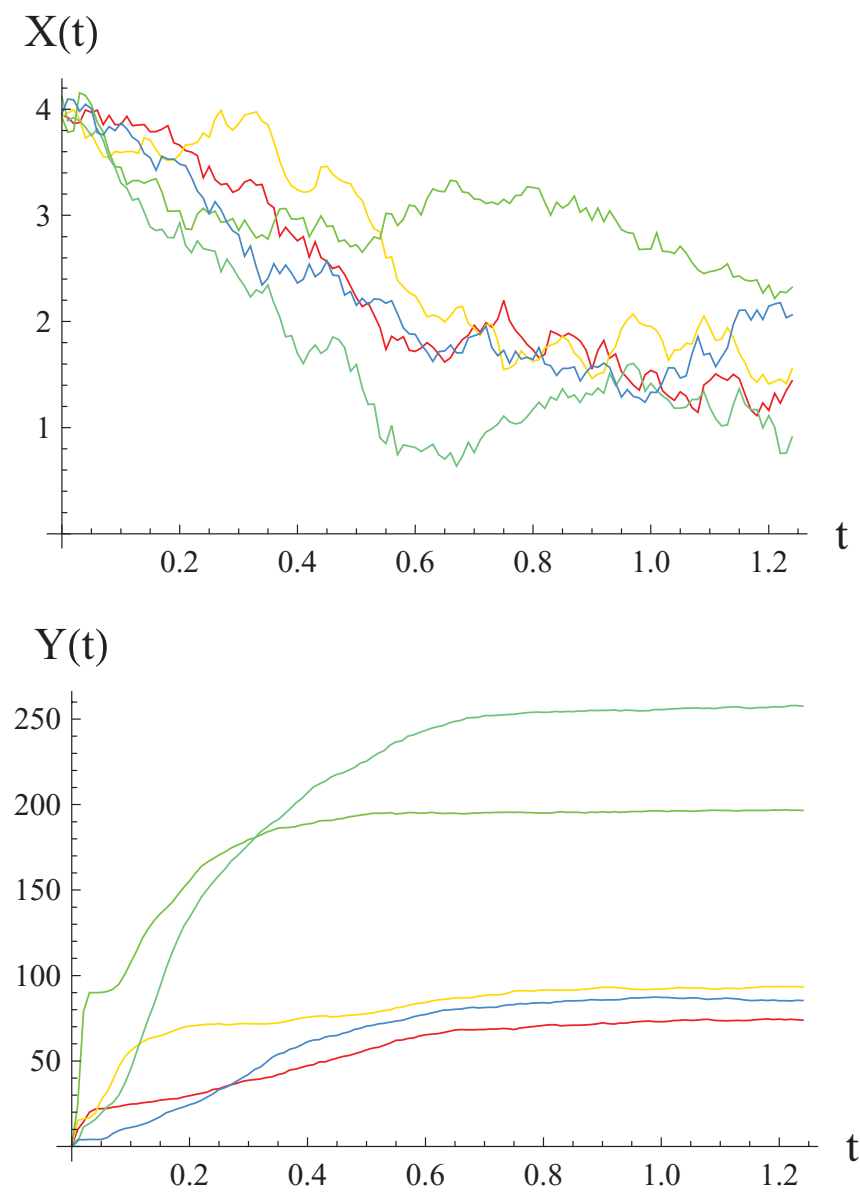

FIG. 3. (Color online) Different numerical realizations of the OU process and the corresponding entropy production Eq. (84). Parameters: $x_{0}=4, D=1, k=1$. 
The velocity and local instantaneous entropy production rate are

$$
v(x, t)=-k x+\frac{k\left(x-x_{0} e^{-k t}\right)}{1-e^{-2 k t}}=\frac{k\left(x-x_{0} e^{k t}\right)}{e^{2 k t}-1}
$$

and

$$
\sigma=\frac{2 k^{2}\left(x-x_{0} e^{k t}\right)^{2}}{D\left(e^{2 k t}-1\right)^{2}} .
$$

Thus, there is a curve $x=x_{0} e^{k t}$, where locally entropy production is zero and trajectories have the same distribution of slopes when looked at forward and backward in time. This is shown in Fig. 2. The results of the entropy balance are plotted in Fig. 3. It is interesting to note how the entropy production rate approaches zero at long time, consistently with the fact that the total entropy produced saturates at equilibrium.

\section{CONCLUSIONS}

We have discussed several aspects of time-reversal of diffusion processes, with special attention to the interpretation of their local properties and their relations to entropy production. In particular, a stochastic differential equation for the instantaneous entropy production rate has been introduced and illustrated in the case of the Ornstein-Uhlenbeck process (Fig. 3).

We have emphasized the elements of the local stochastic dynamics responsible for entropy production and time irreversibility. Limiting our considerations to $1 \mathrm{D}$ makes the derivation more transparent and shows the main differences between equilibrium and nonequilibrium steady states due to nonzero velocity current. Recent developments have emphasized how entropy production can be usefully separated into a nonequilibrium steady state and transient contribution [40,56-58], using the NESS current as a reference. Our analysis allows a local view of such contributions through the term $v(x, t)-v_{\mathrm{NESS}}(x)$.

The general expressions allowed by the 1D context also show the difference between the equilibrium potential $\Phi(x)$ and the NESS potential $\Psi(x)$ [cf. Eqs. (18) and (24)], where the first one can be obtained by simple integration of the kinetic drift $h(x)$, while the second one requires a double integration including the probability current. Such a point was previously pointed out for the general $n$-dimensional case $[59,60]$ and is related to a decomposition of the drift into a reversible and irreversible part around NESS [similar to Eq. (86)], which is in turn a form of Helmholtz decomposition of a velocity field [61] (see also Refs. [62-64]). In future works we will further elaborate on this for multidimensional diffusion processes, processes with jumps, and controlled diffusions that bridge between prescribed initial and final conditions.

\section{ACKNOWLEDGMENTS}

We gratefully acknowledge useful discussions with F. Laio, L. Rey-Bellet, J. R. Rigby, and U. Seifert. The suggestions of an anonymous reviewer are also gratefully acknowledged.
[1] D. R. Cox and H. D. Miller, The Theory of Stochastic Processes (Methuen, London, 1965).

[2] C. Gardiner, Handbook of Stochastic Processes 3rd ed. (Springer-Verlag, Berlin, 2003).

[3] H. Risken, The Fokker-Plank Equation, 2nd ed. (SpringerVerlag, Berlin, 1989).

[4] S. M. Ross, Introduction to Probability Models, 3rd ed. (Academic Press, San Diego, 1985).

[5] B. Oksendal, Stochastic Differential Equations, 6th ed. (Springer, Berlin, 2003).

[6] J. Kurkan, J. Phys. A 31, 3719 (1998).

[7] J. L. Lebowitz and H. Spohn, J. Stat. Phys. 95, 333 (1999).

[8] C. Maes, F. Redig, and A. Van Moffaert, J. Math. Phys. 41, 1528 (2000).

[9] H. Qian, Phys. Rev. E 65, 016102 (2001).

[10] P. Gaspard, J. Stat. Phys. 117, 599 (2004).

[11] C. Bustamante, J. Liphardt, and F. Ritort, Phys. Today 58, 43 (2005).

[12] U. Seifert, Phys. Rev. Lett. 95, 040602 (2005).

[13] V. Y. Chernyak, M. Chertkov, and C. Jarzynski, J. Stat. Mech. (2006) P08001.

[14] R. Kawai, J. M. R. Parrondo, and C. Van den Broeck, Phys. Rev. Lett. 98, 080602 (2007).

[15] A. Porporato, J. R. Rigby, and E. Daly, Phys. Rev. Lett. 98, 094101 (2007).

[16] B. D. O. Anderson, Stoc. Process. Appl. 12, 313 (1982).
[17] M. Pavon and F. Ticozzi, J. Math. Phys. 47, 063301 (2006).

[18] D. J. Thomson, J. Fluid Mech. 180, 529 (1987).

[19] G. N. Milstein, J. G. M. Schoenmakers, and V. Spokoiny, Bernoulli 10, 281 (2004).

[20] A. J. Majda and P. R. Kramer, Phys. Rep. 314, 238 (1999).

[21] G. Falkovich, K. Gawedzki, and M. Vergassola, Rev. Mod. Phys. 73, 913 (2001).

[22] B. C. Levy and A. J. Krener, J. Math. Phys. 34, 1846 (1993).

[23] P. Blanchard and P. Garbaczewski, Phys. Rev. E 49, 3815 (1994).

[24] S. Bernstein, in Vehr. des Intern. Matematikerongr., Zurich (1932), Band 1.

[25] E. Schrodinger, Ann. Inst. Henri Poincare 2, 269 (1932).

[26] E. Nelson, Phys. Rev. 150, 1079 (1966).

[27] E. Nelson, Dynamical Theories of Brownian Motion (Princeton University, Princeton, 1967).

[28] B. Jamison, Z. Wahrsch. Verw. Geb. 30, 65 (1974).

[29] F. Guerra, Phys. Rep. 77, 263 (1981).

[30] P. Garbaczewski and R. Olkiewicz, Phys. Rev. A 51, 3445 (1995).

[31] M. Nagasawa, Stochastic Processes in Quantum Physics (Birkhäuser, Basel, 2000).

[32] S. B. Pope and E. S. C. Ching, Phys. Fluids A 5, 1529 (1993).

[33] P. Hanggi, Helv. Phys. Acta 51, 183 (1978).

[34] Y. L. Klimontovich, Physica A 163, 515 (1990).

[35] B. Lindner, New J. Phys. 9, 136 (2007). 
[36] R. Landauer, J. Stat. Phys. 53, 233 (1988).

[37] G. Stolovitzky, Phys. Lett. A 241, 240 (1998).

[38] A. W. C. Lau and T. C. Lubensky, Phys. Rev. E 76, 011123 (2007).

[39] R. van Zon and E. D. G. Cohen, Phys. Rev. E 67, 04610 (2003).

[40] T. Speck and U. Seifert, Europhys. Lett. 74, 391 (2006).

[41] C. Maes, K. Netocny, and B. Wynants, Physica A 387, 2675 (2008).

[42] H. Follmer, Lect. Notes Math. 1158, 119 (1986).

[43] E. Pardoux and P. Potter, Probab. Theory Relat. Fields 76, 15 (1987).

[44] A. Friedman, Stochastic Differential Equations and Applications, Vol. 1 (Academic Press, New York, 1975).

[45] P. E. Kloeden and E. Platen, Numerical Solution of Stochastic Differential Equations, Vol. 23 of Applications of Mathematics: Stochastic Modelling and Applied Probability (Springer-Verlag, Berlin, 1992).

[46] G. Stolovitzky and E. S. C. Ching, Phys. Lett. A 255, 11 (1999).

[47] E. Racca and A. Porporato, Phys. Rev. E 71, 027101 (2005).

[48] I. M. Sokolov, Phys. Rev. E 60, 3402 (1999).
[49] R. Landauer, Phys. Rev. A 12, 636 (1978).

[50] N. G. van Kampen, IBM J. Res. Dev. 32, 107 (1988).

[51] M. Buttiker, Z. Phys. B 68, 161 (1988).

[52] H. Brenner, Phys. Rev. E 72, 061201 (2005).

[53] D. J. Evans and D. J. Searles, Phys. Rev. E 50, 1645 (1994).

[54] G. Gallavotti and E. G. D. Cohen, Phys. Rev. Lett. 74, 2694 (1995).

[55] D. J. Evans and D. J. Searles, Adv. Phys. 51, 1529 (2002).

[56] T. Hatano and S. I. Sasa, Phys. Rev. Lett. 86, 3463 (2001).

[57] M. Esposito and C. Van den Broeck, Phys. Rev. Lett. 104, 090601 (2010).

[58] C. Van den Broeck and M. Esposito, Phys. Rev. E 82, 011144 (2010).

[59] R. Graham, Z. Phys. B 243, 289 (1971).

[60] R. Graham, Z. Phys. B 40, 149 (1980).

[61] J. Xing, J. Phys. A 43, 375003 (2010).

[62] G. L. Eyink, J. L. Lebowitz, and H. Spohn, J. Stat. Phys. 83, 385 (1996).

[63] P. Ao, J. Phys. A 37, L25 (2004).

[64] R. K. P. Zia and B. Schmittmann, J. Stat. Mech. (2007) P07012. 